\title{
The Development of a Semtex-H Simulant for Terahertz Spectroscopy
}

\author{
N. Greenall ${ }^{1}$ (D) - A. Valavanis ${ }^{1}$ - H. J. Desai ${ }^{2}$ D. O. Acheampong ${ }^{2} \cdot$ L. H. Li ${ }^{1}$ • \\ J. E. Cunningham ${ }^{1}$ • A. G. Davies ${ }^{1}$ - E. H. Linfield ${ }^{1}$ • A. D. Burnett ${ }^{1,3}$
}

Received: 14 September 2016 / Accepted: 11 November $2016 /$

Published online: 29 November 2016

(C) The Author(s) 2016. This article is published with open access at Springerlink.com

\begin{abstract}
The development and use of terahertz (THz) frequency spectroscopy systems for security screening has shown an increased growth over the past 15 years. In order to test these systems in real-world situations, safe simulants of illicit materials, such as Semtex-H, are required. Ideally, simulants should mimic key features of the material of interest, such that they at least resemble or even appear indistinguishable from the materials of interest to the interrogating technique(s), whilst not having hazardous or illicit properties. An ideal simulant should have similar physical properties (malleability, density, surface energy and volatility to the material of interest); be non-toxic and easy to clean and decontaminate from surfaces; be recyclable or disposable; and be useable in a public environment. Here, we present a method for developing such an explosive simulant (for Semtex-H) based on a database of $\mathrm{THz}$ spectra of common organic molecules, and the use of a genetic algorithm to select a mixture of compounds automatically to form such a simulant. Whilst we focus on a security application, this work could be applied to various other contexts, where the material of interest is dangerous, impractical or costly. We propose four mixtures that could then be used to test the spectral response of any instrument, working at terahertz frequencies, without the need for an explosive substance.
\end{abstract}

Keywords Terahertz $\cdot$ Semtex $\cdot$ Genetic algorithm $\cdot$ Explosive $\cdot$ Simulant $\cdot$ Time-domain spectroscopy

Electronic supplementary material The online version of this article (doi:10.1007/s10762-016-0336-z) contains supplementary material, which is available to authorized users.

A. D. Burnett

A.D.Burnett@Leeds.ac.uk

1 School of Electronic and Electrical Engineering, University of Leeds, Leeds LS2 9JT, UK

2 Centre for Applied Science and Technology, St Albans, Hertfordshire, St. Albans AL4 9HQ, UK

3 School of Chemistry, University of Leeds, Leeds LS2 9JT, UK 


\section{Introduction}

Terahertz (THz) (300 GHz-10 THz) frequency spectroscopy has been shown to be a useful technique for the sensing, imaging and spectroscopic analysis of a range of explosives [1-5]. A number of high explosive compounds show spectral features (often at frequencies above $1 \mathrm{THz}$ ) that can be used to identify their presence uniquely [2]. Conversely, a number of the non-explosive compounds that are found in composite plastic explosives (such as Semtex-H) show little or no $\mathrm{THz}$ absorption [1]. The $\mathrm{THz}$ absorption spectra of these composites are therefore dominated by the spectral features of their constituent explosives, making their spectral identification relatively simple compared to other illicit compounds such as realworld drugs-of-abuse, which contain a range of common adulterants which also show a number of sharp spectral features [6]. Furthermore, THz radiation is able to penetrate a number of common packaging [2] and clothing materials [7], whilst being non-ionising [8-10]. However, in order to determine the practical applicability of THz-frequency spectroscopy in the real world, rather than laboratory conditions, a non-explosive simulant is required. In this case, a simulant is defined as a substance or formulation which mimics key spectral features of the material of interest to interrogating technique(s), but does not have the hazardous or illicit features. Commercial simulants are available for other detection techniques but these either mimic the elemental content or physical properties [11] of an explosive or contain only trace amounts of the explosive [12]. Neither of these methods would be suitable for simulants at $\mathrm{THz}$ frequencies as they would not reproduce the spectral features used to identify the explosive. Several compounds have been suggested as simulants suitable for THz spectroscopy including L-tartaric acid and glucose [13, 14]; however, neither of these compounds reproduce the spectral features of the explosives well.

The majority of currently available commercial millimetre wave and THz security scanners work in a reflection geometry at frequencies much lower than $500 \mathrm{GHz}$, where no characteristic spectral features exist. In this paper, we will develop a simulant for Semtex-H that is suitable for frequencies higher than $500 \mathrm{GHz}$ which reproduces the relative spectral intensities of the spectral features seen in the THz spectrum of Semtex-H. To do this, we have developed a small library of $\mathrm{THz}$ spectra of common organic compounds. We demonstrate how this library can be used, in conjunction with a spectral selection process based on genetic algorithms, to select a suitable mixture of materials to act as a $\mathrm{THz}$ simulant for Semtex-H automatically. We then experimentally verify our simulants using $\mathrm{THz}$ time-domain spectroscopy (THz-TDS) and compare them to the target spectra. In this case, the target spectrum will be a normalised absorption spectrum of Semtex-H. The normalisation gives preference to the frequency of spectral features and their relative, rather than absolute, intensity. We note that our method described not only pertains to explosive simulants, but could also be used more widely, and may be particularly useful to mimic toxic or costly materials, as long as a suitable library of compound spectra is available.

\section{Experimental}

THz spectra were acquired using two broadband ( $300 \mathrm{GHz}-6 \mathrm{THz}$ ) THz-TDS systems each driven by one of two ultra-fast ( $<20 \mathrm{fs}$ FWHM) Ti/sapphire laser systems [1]; the first used a Femtosource compact (Femtosource), and the second a Vitatra-HP (Coherent) laser. In both systems, the output beam was split into a pump beam $(\sim 90 \%)$ and a probe beam $(\sim 10 \%)$ by a 
beam splitter. THz radiation was generated by focusing the pump beam onto the gap between the two vacuum-deposited $\mathrm{Ti} / \mathrm{Au}$ electrodes of a low-temperature-grown GaAs (LT-GaAs) photoconductive emitter. A 7-kHz AC square wave was used to bias the emitter, and also used as a reference frequency for lock-in detection. To avoid absorption and dispersion in the undoped GaAs substrate, and to observe the high-frequency components more efficiently, the $\mathrm{THz}$ radiation generated from the emitter was collected in a backwards geometry $[15,16]$ (i.e. from the same surface of the emitter that is excited by the laser) and then focused by a pair of off-axis parabolic mirrors onto the sample. A second pair of off-axis parabolic mirrors collected the transmitted THz radiation and focused it onto a $150-\mu \mathrm{m}$-thick GaP electrooptic crystal, mounted collinearly with a near-infrared probe beam for detection. The electric field comprising the $\mathrm{THz}$ waveform was then measured in the time-domain using electro-optic sampling and a lock-in detection scheme [15].

\subsection{Terahertz Spectra of Plastic Explosives}

Semtex-H contains two explosive crystalline compounds, cyclotrimethylenetrinitramine (RDX) and pentaerythritol tetranitrate (PETN), whose individual THz spectra are shown in Fig. 1, along with a number of polymers, plasticisers and anti-oxidants, which we previously showed had no significant THz absorption in the $300-\mathrm{GHz}$ to $5.5-\mathrm{THz}$ range [1]. A viable simulant for Semtex-H must reproduce spectral peaks at similar frequencies to the five main peaks in the Semtex-H spectrum $(0.79,1.44,1.98,2.15$ and $2.92 \mathrm{THz})$, and have similar relative intensities. This poses a key challenge since the absorption peaks are not simply related to the presence of specific functional groups (as is the case in mid-infrared spectroscopy), but instead are mainly attributed to translatory and rotary external modes, which are affected not just by the molecular structure, but also by the crystalline structure of the material $[17,18]$. The origin of the modes in both RDX [19, 20] and PETN [17, 21-25] is relativity well understood at THz frequencies, however. We note that in principle it would be possible to modify the structure of RDX and PETN to form isomorphous crystals that are non-explosive [26], whilst at the same time minimising the change to their $\mathrm{THz}$ spectra. This would represent a significant challenge in chemical synthesis. A more promising route, taken here, is to find spectra of simple organic compounds, preferably with only a few peaks in this spectral range, and to then combine them to form a composite spectrum similar to that of Semtex-H. The simulant, at least in the first instance, needs to replicate both the general shape and relative intensity of the spectral features in the absorption spectra of Semtex-H. It, however, does not need to perfectly mirror these features, but rather, it is the relative change across the frequency window that is important in order to test and calibrate equipment without using a hazardous compound.

A wide range of organic crystalline compounds which exhibit spectral features in the 1- to 6$\mathrm{THz}$ band can potentially be used to simulate the spectrum of Semtex-H. However, only a small number of solid compounds have been found to have spectral features below $1 \mathrm{THz}$ reproducing the $0.79-\mathrm{THz}$ peak of RDX (Fig. 1). This spectral region is therefore the most difficult to represent by a simulant, and it is likely that a compromise in mixture will be made in order match this spectral feature, even if this is to the detriment of the match in other spectral regions.

\subsection{Development of a Sample Database}

In order to determine compounds that could be included in the simulant mixture, the spectra of a range of sugars and other commonly available organic compounds were measured (Fig. 2), 
Fig. 1 THz spectra of RDX (black) PETN (red) and Semtex-H (blue). Both the RDX and PETN samples are $20 \% \mathrm{w} / \mathrm{w}$ concentration mixed with PTFE as a THztransparent matrix material [1]. The spectra of PETN and Semtex$\mathrm{H}$ have both been offset by 5 and $10 \mathrm{~mm}^{-1}$ respectively for clarity

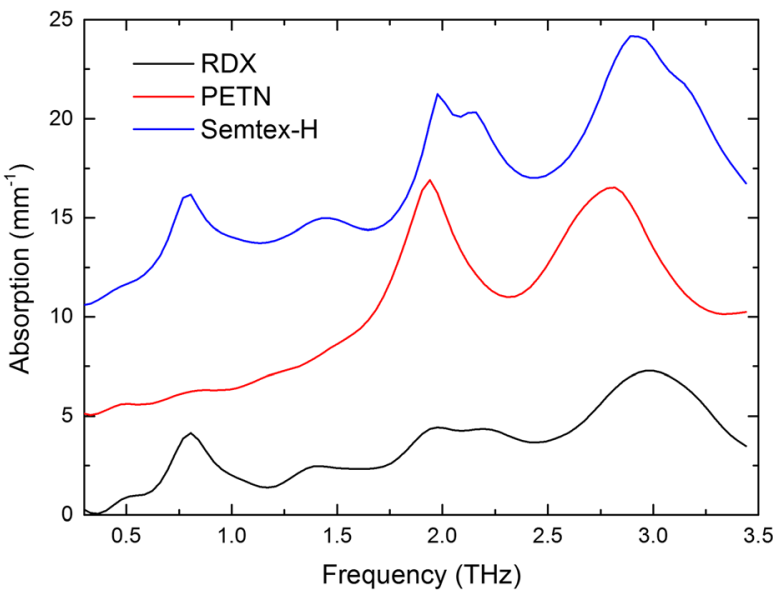

which between them have a wide range of spectral parameters. Having a large number of components increases the number of possible solutions, and therefore the chance of a good solution. This increase in possible solutions, however, also makes the selection of an optimal solution more difficult. A range of non-explosive compounds that are chemically similar to known explosives were also measured: hexamethylenetetramine and cyanuric acid, which have a similar heterocyclic ring system to RDX; erythritol and pentaerythritol, which are similar to PETN and artemisinin, which contains an unusual peroxide bridge (as do some common primary explosives such as triacetone triperoxide (TATP) and hexamethylene triperoxide diamine (HMTD)). The resultant spectra for these samples are presented in Fig. 2d. The 16 compounds considered in Fig. 2 were used to form a library of spectra, from which Semtex-H simulants were designed. A number of the samples shown in Fig. 2 are not pure but were diluted using a THz-transparent matrix material (PTFE) in order to obtain spectra over the full dynamic range of the THz-TDS system.

\section{Results and Discussions}

In order to create a simulant from a mixture of compounds, we modelled the spectra of a mixture, and then quantified the similarity of the resultant spectra to the target spectra (the normalised spectra of Semtex-H). As many of the samples in the library are diluted, it is important to estimate the total absorption of the pure compound; thus, it was assumed that the Beer-Lambert law applies, and that the measured relative absorption coefficient could be multiplied by the mass ratio of the diluted compound to obtain the absorption coefficient of the 'pure material'. These 'pure' spectra from each compound were then used to form a library of spectra. In a similar fashion, to model the spectra of a mixture, a weighted sum of the constituent 'pure' THz spectra was computed over the required frequency range to approximate the spectrum of a mixture $M_{N}$ :

$$
M_{N}(\omega)=\sum_{i=1}^{N} C_{i} S_{i}(\omega)
$$

where $N$ is the number of components, $S_{i}$ the 'pure' THz absorption spectrum of each component $i$ taken from the library of spectra and $C_{i}$ the concentration (normalised to one) of each 

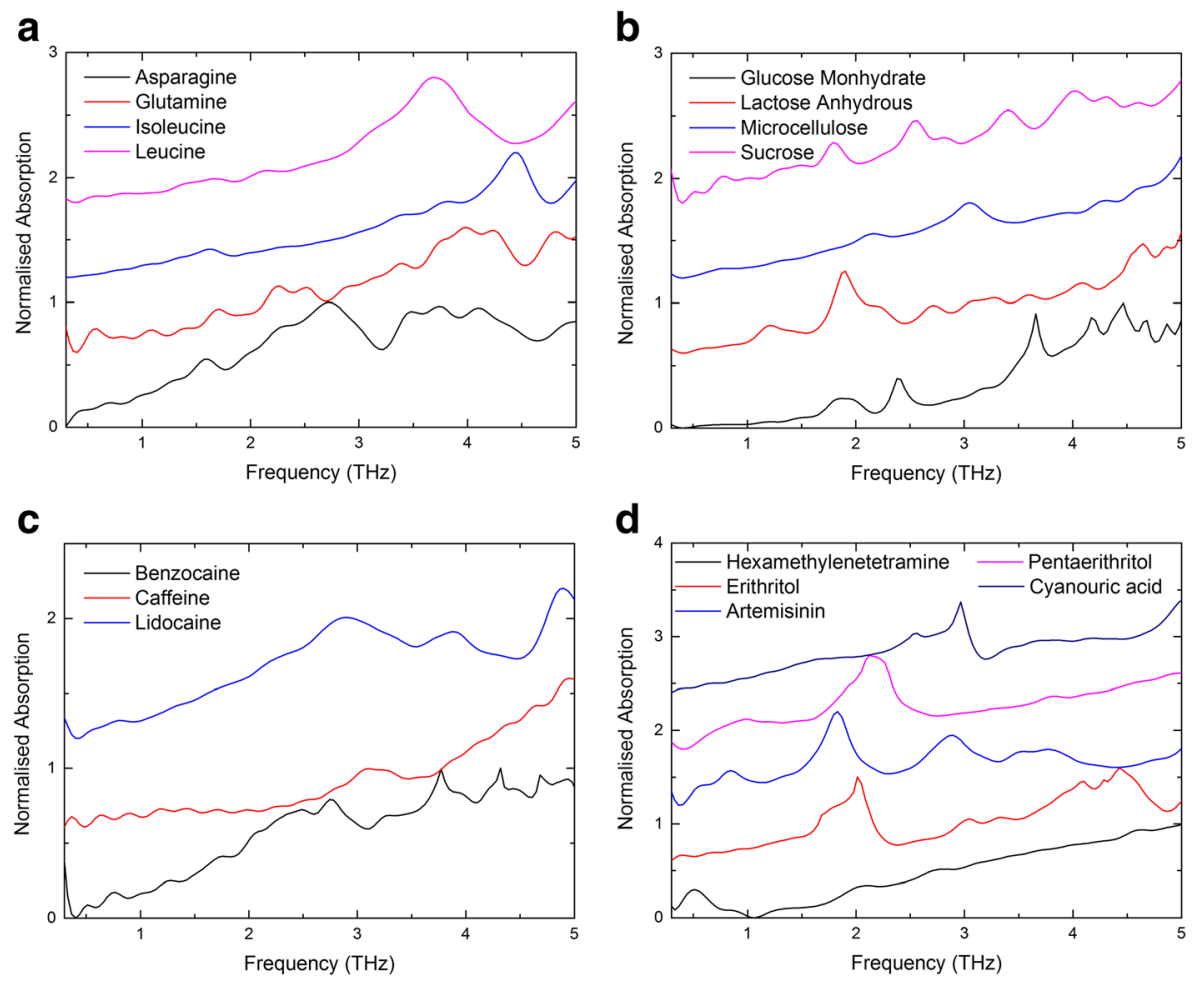

Fig. 2 THz spectra of a range of organic molecules including a amino acids, $\mathbf{b}$ sugars and carbohydrates, $\mathbf{c}$ a range of organic compounds that have been considered as drug adulterants [6] and $\mathbf{d}$ non-explosive compounds that are chemically similar to known explosives. All spectra were normalised to have a maximum absorption of one, and are offset for clarity. The original, non-normalised data is available as part of the dataset available at the Leeds Data Repository which is linked to this article [28]

component by mass, and frequency $\omega$. Whilst this model is simple, it is sufficient to find a viable simulant mixture.

Once a viable mixture is identified, its spectra are compared to the target spectra, using a function to quantise the similarity. We have chosen to use cross-correlation as the basis for this function. Cross-correlation is a well-known measure of similarity [27], and can be thought of as a method to quantise the overlap between spectra. This operation will shift the two spectra relative to each other, to change the shared area, thus giving a score of similarity as a function of relative frequency shift:

$$
C_{A B}(r)=\sum_{n=0}^{n=N} A(n) B(n+r)
$$

where $r$ is the frequency shift between the spectra's $A$ and $B, N$ is the number of samples in each of the spectra, $n$ is the frequency index and $C$ is the cross-correlation. We use this frequency shift to provide our similarity scores with a degree of frequency tolerance. Another approach we considered was to minimise the difference (sometimes referred to as deviation of error) between the two spectra. We have found that this produces featureless simulant spectra which minimises the average difference across the entire spectrum. We find cross-correlation to be a more suitable 
measure of similarity, which agrees with previous literature on the subject [24]. We use a weighted normalised correlation coefficient to provide a normalised similarity score between 0 and 1 [27]:

$$
C_{A B}^{W s}=\frac{\sum_{r=-l}^{r=l} C_{A B}(r) W(r)}{\sqrt{\sum_{r=-l}^{r=l} C_{A A}(r) W(r)} \sqrt{\sum_{r=-l}^{r=l} C_{B B}(r) W(r)}}
$$

where $W(r)$ is a weight function, defined as:

$$
W(r)=1-\frac{|r|}{l}
$$

Here, $l$ is what we term a frequency shift tolerance, i.e. the absolute shift of a spectral feature that will be tolerated by the fitness function before a discriminating penalty is applied. The weight function is used to introduce a preferential score bias to small frequency shifts. A simplified schematic of how this was used can be seen in Fig. 3. In Fig. 3a, the two spectral features are identical in spectra $\mathrm{A}$ and $\mathrm{B}$ but shifted by a value $r$. As $r$ is greater than $l$, the weighting function is reduced and the final score is poor. In Fig. 3b, $r$ is less than $l$ and the spectral feature in each spectra is identical; however, $r$ is still quite large. In Fig. 3c, $r$ is smaller than in Fig. $3 b$ but the spectral features are not identical. The spectra in Fig. 3c will always give a better score than Fig. $3 b$.

$C_{A B}^{W S}$ gives a score between 1 (spectra are identical) and 0 (spectra are completely different). Thus, a mixture of compounds that form a suitable Semtex-H simulant must give a resultant spectrum with a high value of $C_{A B}^{W S}$. However, with even a modest number of compounds, a singular optimal solution is difficult to obtain, because the number of degrees of freedom in the computation is equal to the number of compounds. Therefore, this problem was solved using a genetic algorithm, which is a random process which considers solutions taken uniformly from all possible solutions. This means the minimisation problem is not used to direct the minima search and can converge on a global solution.

\subsection{A Genetic Algorithm for Developing Simulant Mixtures}

Genetic algorithms are a class of minimiser/maximiser algorithms based on the principles of evolution [29]. A genetic algorithm is an iterative process which works on a population of

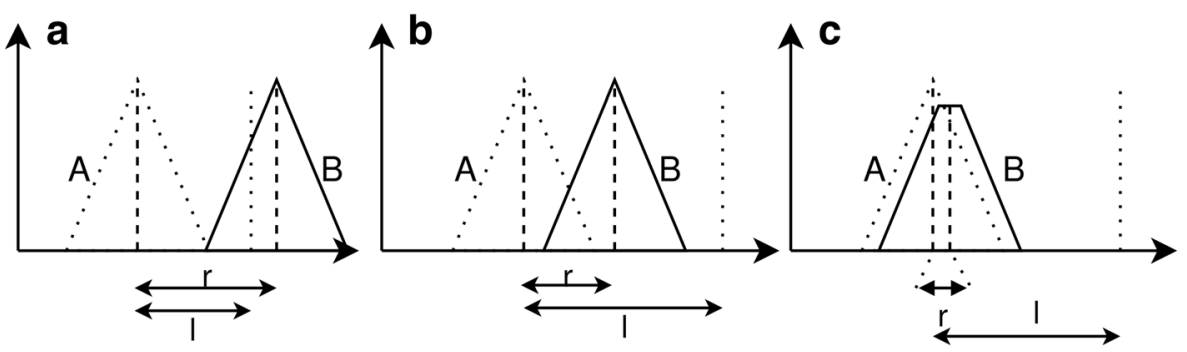

Fig. 3 A diagram to illustrate how spectra is evaluated in relation to frequency difference $(r)$ between the target feature $(A$, dotted $)$ and simulant feature $(B$, solid $)$, and frequency tolerance $(l)$. a $r$ is greater than 1 , the crosscorrelation is truncated using $W(r)$, giving a poor score. b The simulant feature has an identical shape, and thus good cross-correlation, but large $r$, giving a poorer score due to $W(r)$ than $\mathbf{c}$, where the simulant feature is only partially similar in shape, but has smaller $r$ 
solutions, which in this case are the predicted mixture spectra. Each solution is evaluated against the target spectrum (the normalised spectrum of Semtex-H) by a fitness function, which provides a fitness score. Poorer scoring solutions are removed from the population and replacements are produced based on modified higher-ranked solutions (see online resource 1 for a detailed description of the genetic algorithm methodology). In this case, the fitness function $F$ is given by:

$$
F=100\left(1-\left|C_{A B}^{W s}\right|\right)
$$

$F$ is, therefore, simply the weighted normalised correlation coefficient $C_{A B}^{W s}$ scaled between 0 and 100, with 0 representing a perfect match for the normalised spectrum of Semtex-H, and 100 representing no correlation. This is done to create a minimisation problem suitable for the genetic algorithm. The genetic algorithm was implemented using MATLAB's global optimisation toolbox [30]; for further information on the implementation, please see the online resource 1 . To create viable simulants, a two-stage process was used: the first being an initial selection of suitable compounds for a mixture, followed by a second stage, an optimisation of the concentrations of each component within the mixture.

For the component-selection stage (stage 1), the genetic algorithm was used to form five compound combinations, with each component making up $20 \%$ of the mixture. Repetitions of each component were allowed within an individual mixture. For instance, if the algorithm generated a mixture spectra formed from a combination of components 'A, B, C, A, and A' for example, this would be converted to a three component mixture containing ' $\mathrm{A}, \mathrm{B}$, and $\mathrm{C}$ ' with concentrations of 60,20 and $20 \%$ by mass, respectively. The initial population of mixture spectra used was uniformly randomly generated. The mixture spectra were then modelled using Eq. 1, and then evaluated against the normalised spectrum of Semtex-H using the fitness function, $F$, in Eq. 5. The population of mixture spectra were then modified with poorer scoring spectra removed and additional spectra generated from the remaining population (see online resource 1). The evaluation, using fitness scores, and modification of the population were repeated until a consistent best-ranked solution appeared, with a change in fitness function less than $1 \times 10^{-6}$ over 50 iterations.

This process (stage 1) of selecting a suitable component combination was repeated a total of 30 times, deliberately making use of the non-deterministic selection process to produce multiple viable solutions. The highest-ranked mixture spectra from each of the 30 repeats were then compared, duplicates were removed and the remaining mixture spectra ranked. The top two scoring solutions were then used as a basis for the second stage, in which the genetic algorithm was used to optimise the concentration of each component of the simulant mixture with the two highest-ranked mixtures, from stage 1, being used to form the initial population. The genetic algorithm was performed in a similar manner to the first stage, but changes were now made to component concentrations for a fixed component selection. These concentrations were then used to form the spectrum of the mixture, which was then compared to the normalised spectra of Semtex-H using the fitness function $F$ (Eq. 5).

As in stage 1 , the process was repeated until a consistent best solution with a change in fitness function of less than $1 \times 10^{-6}$ occurred over 50 iterations was achieved. This second stage was then repeated a further four times, and the mean concentrations from each run used as the concentration for the final solution. A detailed account of the method used is included within online resource 1 , including flow diagrams of both stages.

The whole process (both initial selection, stage 1, and concentration optimisation, stage 2) was then repeated ten times, over eight different frequency tolerances ( $l$ ) (see Eqs. 3 and 4), and the final solution to each run collated. Over all these repeats, four common mixtures were 
produced. This full process takes roughly 3 hours running on a single core, and could be optimised significantly using parallel processing techniques.

Table 1 shows the components, concentrations and fitness function score $(F$, where 0 is a perfect match) for each of the four unique mixtures that were obtained using the genetic optimisation algorithm. The process converged on similar mixtures over different frequency tolerances, where components were the same, with preference being given to the mixture at the lowest frequency tolerance. Three mixtures were obtained using the lowest frequency tolerance of $37 \mathrm{GHz}$ (mixtures 1, 2 and 3 in Table 1). A further solution (mixture 4) was produced once the frequency tolerance was increased significantly to $620 \mathrm{GHz}$, but which concomitantly resulted in a much lower fitness function score. This lower score is because a greater frequency shift tolerance weakens the constraint of matching the frequency of features in the target spectra, which can give a better fitness score and a greater diversity of solutions, at the expense of matching target feature location in frequency. In the case of mixture 4, the feature at $1.7 \mathrm{THz}$ is producing a good match with Semtex-H's doublet at $\sim 2 \mathrm{THz}$ because of the greater frequency shift tolerance allowed in this case. The fitness score for mixtures obtained using different frequency tolerances are not directly comparable. We have found, however, that we did not need to increase frequency shift tolerance to achieve multiple low fitness solutions. This is unsurprising given the large full-width-at-half-maximum of the majority of the spectral features being imitated. All four mixtures contain artemisinin, which closely simulates the lowfrequency spectral feature of RDX $(0.79 \mathrm{THz})$ along with a number of higher-frequency spectral features of both RDX and PETN. Microcellulose was also a significant component of three of the mixtures, as it usefully provided a weak contribution to peaks at similar frequencies to those in Semtex-H at $0.79,1.44,1.98,2.15$ and $2.92 \mathrm{THz}$, as well as a background that increases with frequency, similar to that seen in Semtex-H.

Given the non-deterministic nature of these algorithms, it is important to consider the consistency of the results. Such a consistency check is typically performed by increasing the population size used in the genetic algorithm, which forces the algorithm to consider a larger and more varied selection, and/or by repeating the process multiple times. The number of components in the first part of the process was deliberately kept low $(<5)$ to minimise the complexity of the resultant mixture in order to make it easier to fabricate. When calculations were performed with a larger number of components (up to 20), it was found that the concentrations of many of the additional components tended to zero in the second stage of the optimisation process, but more importantly, the new mixtures scored no better in the fitness function than those developed

Table 1 The four algorithm-generated mixtures, their components, mass percentages and the fitness function value of the spectra compared to the normalised spectrum of Semtex-H

\begin{tabular}{|c|c|c|c|}
\hline Mixture number & Component name (concentration) & $\begin{array}{l}\text { Fitness function, } \\
F(0-100)\end{array}$ & $\begin{array}{l}\text { Frequency } \\
\text { tolerance }(\mathrm{THz})\end{array}$ \\
\hline 1 & Artemisinin $(21 \%)$, microcellulose $(79 \%)$ & 1.88 & 0.037 \\
\hline 2 & $\begin{array}{l}\text { Artemisinin }(18 \%), \text { microcellulose }(64 \%) \text {, } \\
\text { hexamethylenetetramine }(18 \%)\end{array}$ & 1.8172 & 0.037 \\
\hline 3 & $\begin{array}{l}\text { Artemisinin }(17 \%) \text {, microcellulose }(51 \%) \text {, } \\
\text { hexamethylenetetramine }(27 \%), \\
\text { asparigine }(5 \%)\end{array}$ & 1.8223 & 0.037 \\
\hline 4 & $\begin{array}{l}\text { Artemisinin }(23 \%) \text {, hexamethylenetetramine }(53 \%) \text {, } \\
\quad \text { lidocaine }(24 \%)\end{array}$ & 0.40923 & 0.62 \\
\hline
\end{tabular}


using the method described above. If we compare to the number of features in the target spectra, the reason for this becomes clear. There are only four broad peaks in the target spectra; therefore, produced simulants will only require a similar number of components to mimic these features.

The resulting predicted spectra for each of the four mixtures are shown as dashed-dotted lines in Fig. 4, with each spectra showing four broad spectral features centred roughly at 0.75 , 1.5, 2 and $3 \mathrm{THz}$. Each mixture was then produced and diluted to $25 \%$ concentration with PTFE before being pressed into a solid pellet. The THz spectra for each of these samples are shown as solid lines in Fig. 4. Figure 4 also shows the normalised spectra of Semtex-H (black solid line) from Fig. 1 with all spectra being offset for clarity and comparison.

The experimentally measured spectra of each of the four mixtures are not identical to the predicted spectra; peaks in the spectra are generally weaker and less prominent. These discrepancies are likely caused by a number of factors. First, we have assumed that the absorption intensity scales linearly with concentration both in the formation of the library, and then in Eq. 1 when forming the mixture spectra. A more advanced method would be to use an effective medium approximation, such as the Maxwell-Garnett approximation [31], to estimate both the permittivity of the 'pure' materials for the library and also to estimate the effect of combining these library spectra in to a mixture. However, this would have significantly increased computation time, without a significant improvement in accuracy for the permittivity, particle sizes and concentrations used here [32].

Secondly, to construct the library, we diluted our samples with a non-absorbing polymer matrix (PTFE) which can cause slight changes in peak positions $[18,33]$ compared to the spectra of the pure material. Finally, mixing a number of substances will certainly affect the THz scattering [34-36], particularly if the refractive indices of the components differ (which is often the case at $\mathrm{THz}$ frequencies), and in addition to increasing the total scattering background, this may also lead to changes in spectral peak shape [35]. This change in scattering may lead to the greatest difference between the predicted and measured spectra, but there is no simple method to account for this.

The measured spectra do show a very high similarity to that of Semtex-H, with Table 2 showing the fitness score for the four measured spectra. Although these fitness scores are poorer than predicted, due to the approximations in predicting mixture spectra described previously and inhomogeneities within the physical mixture, the four powdered samples are all suitable simulants for Semtex-H, showing broad spectral features at similar frequencies and

Fig. 4 The four predicted spectra for the simulant mixtures are shown as dashed-dotted lines. The solid lines show the measured spectra of the mixtures once they were made, diluted with PTFE to $25 \%$ by mass and pressed into solid pellets. The solid black line shows the normalised spectra of Semtex-H previously shown in Fig. 1 for comparison. Spectra have been normalised and offset for clarity

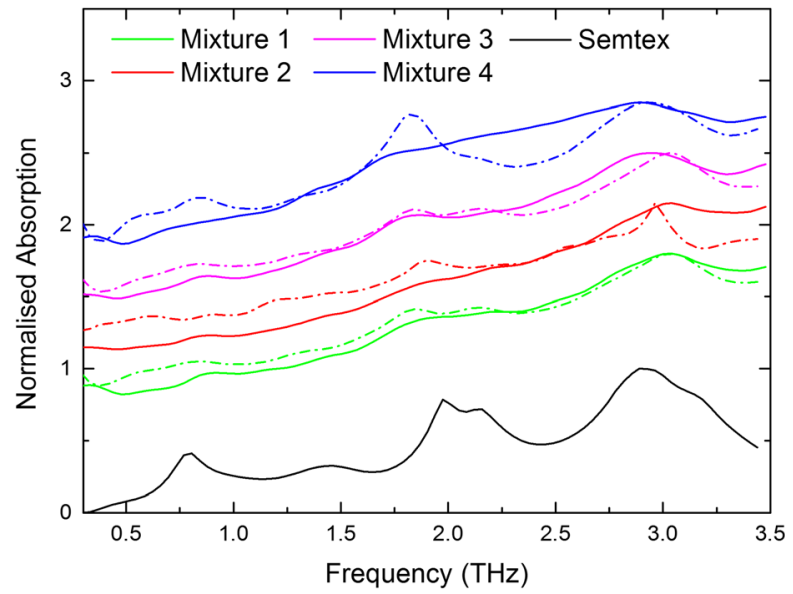


Table 2 The fitness function score $(F)$ for the measured spectra of the four mixtures, compared to the spectra of Semtex-H
Sample number

Fitness function

1

4.5039

2

6.8988

3

4.8832

4

with similar absorption ratios to those of the $\mathrm{THz}$ spectrum of Semtex-H. In order to determine why these four mixtures were chosen by the genetic algorithm discussed above, we broke the contributions to the spectra down using mixture 1 as a test case, as it was the simplest mixture produced and has components common to almost all of the other mixtures.

Figure 5 shows the normalised, baseline corrected, spectra (solid lines) for both Semtex-H (black) and the predicted spectra mixture 1 (red). Both spectra were baseline corrected using an asymmetric least squares smoothing method [37] with the baselines shown as dotted lines in Fig. 5. The algorithm is over-estimating the spectral background by choosing materials that have a large spectral background such as microcellulose; this in-turn reduces the spectral heights of each feature. Each spectral feature is, however, reproduced in some form and the relative intensities (Fig. 5b) of each feature are similar in both spectra.

In order to analyse the mixture fitness relative to frequency, we have developed a method to determine the frequency-dependent fitness function using a sliding window and used this to compare the normalised spectra of Semtex-H and mixture 1. Here, we calculated the fitness function within a short sliding window, moved along the frequency axis in $37 \mathrm{GHz}$ steps. A $320-\mathrm{GHz}$ window was chosen as it was the largest window which would distinguish the individual peaks of the central doublet in the mixture spectra; adjusting the window size will change the effective resolution of the sliding window fitness function, the minimum width being the frequency resolution of the instrument. The results of this comparison can be seen in Fig. 6. The top pane of Fig. 6 shows the normalised spectra of both Semtex-H (black) and mixture 1 (red) with the grey box indicating the window used to calculate the fitness function at $1.83 \mathrm{THz}$. The lower pane shows the frequency-dependent fitness function with the green shading in the top pane showing this same data normalised in order to emphasise the areas of

a

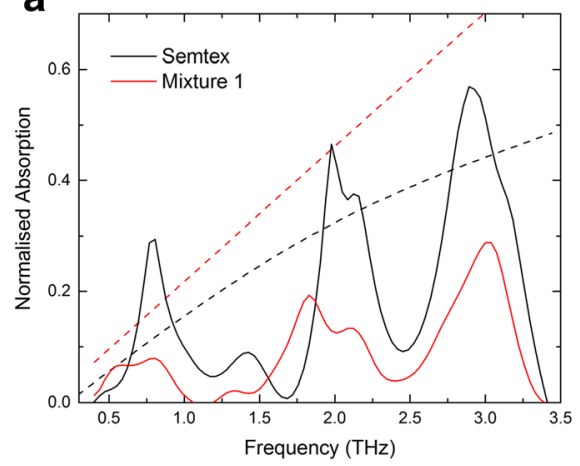

b

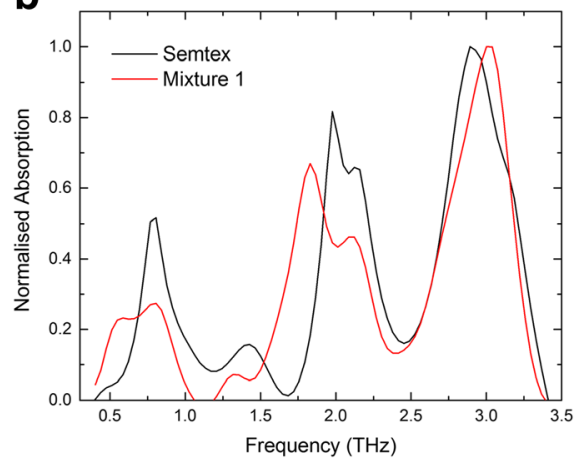

Fig. 5 a The normalised baseline corrected absorption spectra (solid lines) of both Semtex-H (black) and the predicted spectra of mixture $1(\mathrm{red})$. Baseline correction was performed using an asymmetric least squares smoothing method [37] and the subtracted baselines are shown as dotted lines for comparison. b The same baseline corrected spectra shown in $\mathbf{a}$, but normalised to allow the relative peak heights to be compared easily 
Fig. 6 The frequency-dependent fitness score calculated using a sliding window. The top pane shows the normalised spectra of both Semtex-H (black) and mixture 1 (red) with the grey box indicating the window used (320 GHz wide) to calculate the fitness function at $1.83 \mathrm{THz}$. The lower pane shows the frequencydependent fitness function (blue) with the green shading in the top pane showing the same data normalised
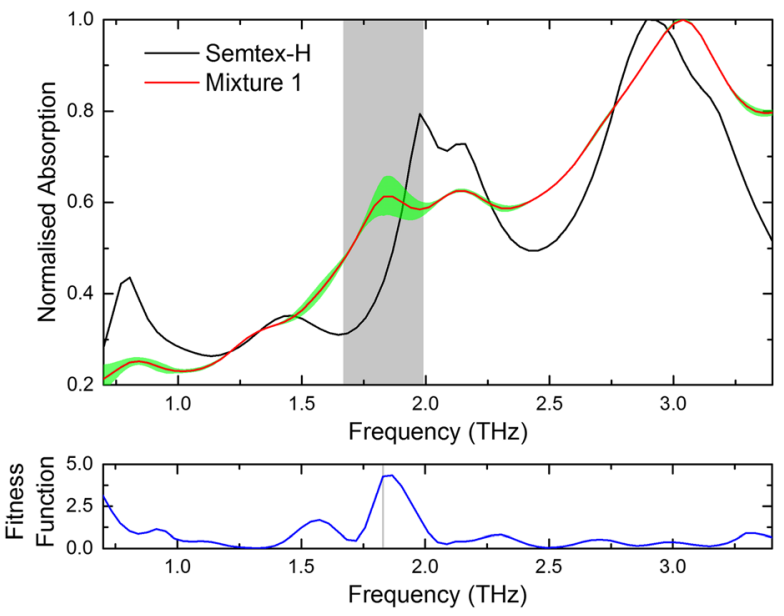

the mixture spectra that show poor correlation. What is clear from data is that it is the poor fit around the feature at $0.79 \mathrm{THz}$ and the doublet at 1.98 and $2.15 \mathrm{THz}$ that governs the resultant mixture. It is artemisinin that contributes to both these features in all four mixtures; increasing its concentration will improve the spectral match at $0.79 \mathrm{THz}$, whilst producing an inferior mixture in comparison to the doublet. Reducing the concentration of artemisinin will have the opposite effect. This compromise between matching the sub-1-THz peak and the doublet governs the choice of mixtures generated by the genetic algorithm, whilst it is the lack of compounds within the literature as a whole with spectral peaks below $1 \mathrm{THz}$ that currently limits the development of a more appropriate mixture at this time.

\subsection{Improving the Physical Properties of the Semtex-H Simulant}

A polycrystalline sample does not have the same physical properties as Semtex-H. If the physical properties could be suitably modified, however, the sample would make a more suitable explosive simulant. We chose to improve the physical properties of mixture 1 to make it closer to those of Semtex-H, owing to its simpler composition. A plastic explosive is simply an explosive compound embedded in a soft polymer matrix that allows the sample to be malleable and handled safely by reducing the overall sensitivity of the explosive compound. To replicate this polymer matrix, polystyrene-co-butadiene pellets were first dissolved, whilst stirring, in dichloromethane. Once the polystyrene-co-butadiene was fully dissolved and the majority of the excess dichloromethane and left to evaporate in ambient conditions, tributyl citrate, n-octyl phthalate and $\mathrm{N}$ phenyl-2-napthylamine were added in a 3:3:3:1 ratio by mass. Once the polymer solution is made, a sample of mixture 1 was then added in a 4:1 ratio by mass, and the resultant mixture left to dry in ambient conditions. This formed a material similar in texture, consistency and density to Semtex$\mathrm{H}$ and resembles other Centre of Applied Science and Technology (CAST) "Hemtex class" compounds $[38,39]$. The spectrum of this polymer-embedded simulant was then compared to those of mixture 1 and Semtex-H (Fig. 7). Small changes in spectrum between mixture 1 and the polymer-embedded sample can be seen, possibly due to the differences in solubility of artemisinin and microcellulose in dichloromethane. We find that the sample retains all of the defining features as a spectral simulant, and that this method of implanting powdered simulants into a polymer is a viable one for the creation of simulants for use at $\mathrm{THz}$ frequencies. 
Fig. 7 The normalised spectrum of Semtex-H (black), Mixture 1 (Red) and the polymer-embedded version of mixture 1 (blue)

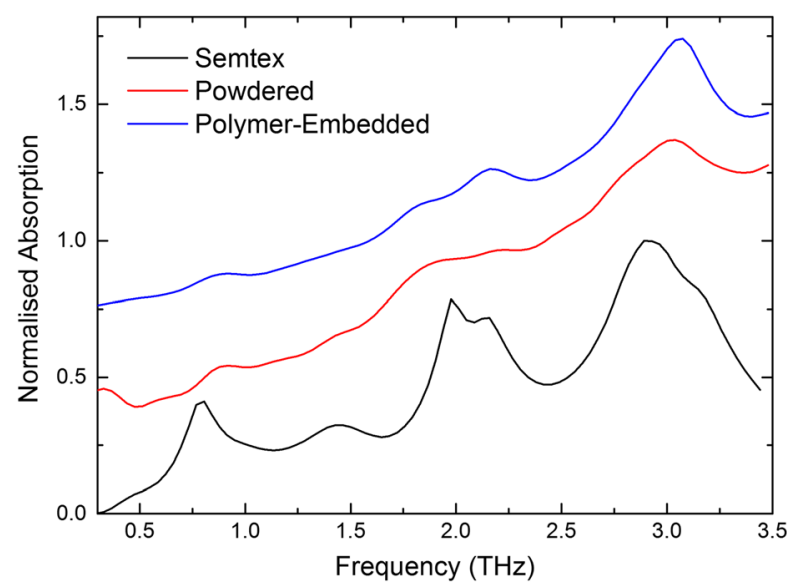

\section{Conclusions}

We have demonstrated a method for automatically selecting a mixture of samples from a library of spectra by using a genetic algorithm to create a simulant for a common high explosive (Semtex-H). Using this method, we have created four mixtures of compounds that have $\mathrm{THz}$ frequency spectra with similar spectral features to this material. Finally, we have embedded the powdered samples in a polymer matrix, to replicate the physical properties of Semtex-H; this sample could be used to test other security systems that rely on physical rather than chemical properties. The methodology developed here could also be used diagnostically, to obtain the constituent spectral components of $\mathrm{THz}$ spectra of a mixture, given a suitably complex database, although methods to understand scattering in mixtures combined with effective medium approximations may be needed to improve the accuracy of our methods. It is also worth noting that the algorithm is not specific to a security context, and could be used for a range of simulants, including those needed to calibrate biomedical diagnostic systems or for pharmaceutical quality testing, without the need for biological samples or costly synthetic compounds.

Acknowledgments This work was supported by the Engineering and Physical Sciences Research Council (EPSRC) Doctoral Training Partnership Grant (to N.G., EP/L504993/1) and EPSRC Postdoctoral Fellowship EP/ I026657/1 (to A.D.B.). Support from the Royal Society, Wolfson Foundation (AGD and EHL) and the Centre for Applied Science and Technology's 'Innovations' initiative is also acknowledged. The data associated with this paper are openly available from the University of Leeds data repository [28] and in the supplementary information accompanying this paper.

Open Access This article is distributed under the terms of the Creative Commons Attribution 4.0 International License (http://creativecommons.org/licenses/by/4.0/), which permits unrestricted use, distribution, and reproduction in any medium, provided you give appropriate credit to the original author(s) and the source, provide a link to the Creative Commons license, and indicate if changes were made.

\section{References}

1. W.H. Fan, A. D. Burnett, P. C. Upadhya, J.E. Cunningham, E. H. Linfield, A. G. Davies: Far-infrared spectroscopic characterization of explosives for security applications using broadband terahertz timedomain spectroscopy. Applied Spectroscopy. 61, 638-643 (2007) 
2. Davies, A.G., Burnett, A.D., Fan, W., Linfield, E.H., Cunningham, J.E.: Terahertz spectroscopy of explosives and drugs. Mater. Today. 11, 18-26 (2008)

3. Kemp, M.C., Taday, P., Cole, B.E., Cluff, J., Fitzgerald, A.J., Tribe, W.R.: Security applications of terahertz technology. AeroSense 2003. 5070, 44-52 (2003)

4. Shen, Y., Lo, T., Taday, P., Cole, B., Tribe, W., Kemp, M.: Detection and identification of explosives using terahertz pulsed spectroscopic imaging. Appl. Phys. Lett. 86, 241116 (2005)

5. Leahy-Hoppa, M., Fitch, M., Zheng, X., Hayden, L., Osiander, R.: Wideband terahertz spectroscopy of explosives. Chem. Phys. Lett. 434, 227-230 (2007)

6. Burnett, A.D., Fan, W., Upadhya, P.C., Cunningham, J.E., Hargreaves, M.D., Munshi, T., et al.: Broadband terahertz time-domain spectroscopy of drugs-of-abuse and the use of principal component analysis. Analyst. 134, 1658-1668 (2009)

7. Baker, C., Lo, T., Tribe, W., Cole, B., Hogbin, M., Kemp, M.: Detection of concealed explosives at a distance using terahertz technology. Proceedings of the IEEE. 95, 1559-1565 (2007)

8. Kleine-Ostmann, T., Jastrow, C., Baaske, K., Heinen, B., Schwerdtfeger, M., Karst, U., et al.: Field exposure and dosimetry in the thz frequency range. Terahertz Science and Technology, IEEE Transactions on. 4, 1225 (2014)

9. Hintzsche, H., Stopper, H.: Effects of terahertz radiation on biological systems. Crit. Rev. Environ. Sci. Technol. 42, 2408-2434 (2012)

10. Berry, E.: Risk perception and safety issues. J. Biol. Phys. 29, 263-267 (2003)

11. Kury, J.W., Anderson, B.L.: Explosive simulants for testing explosive detection systems. US5958299A, (1999)

12. Simpson, R.L., Pruneda, C.O.: Non-detonable and non-explosive explosive simulators. US5648636A, (1997)

13. Palka, N.: THz reflection spectroscopy of explosives measured by time domain spectroscopy. Acta Physica Polonica A. 120, 713-715 (2011)

14. Konek, C., Wilkinson, J., Esenturk, O., Heilweil, E., Kemp, M.: Terahertz spectroscopy of explosives and simulants: RDX, PETN, sugar, and L-tartaric acid. 7311, 73110K-73110K-73117 (2009)

15. Shen, Y., Upadhya, P., Linfield, E., Beere, H., Davies, A.: Ultrabroadband terahertz radiation from lowtemperature-grown GaAs photoconductive emitters. Appl. Phys. Lett. 83, 3117-3119 (2003)

16. Upadhya, P., Fan, W., Burnett, A., Shen, Y., Beere, H., Davies, A., et al.: Ultra-broadband coherent terahertz spectroscopy using asymmetric excitation of photoconductive structures. IRMMW-THz 2005. 2, 451-452 (2005)

17. Burnett, A.D., Kendrick, J., Cunningham, J.E., Hargreaves, M.D., Munshi, T., Edwards, H.G., et al.: Calculation and measurement of terahertz active normal modes in crystalline PETN. ChemPhysChem. 11, 368-378 (2010)

18. Burnett, A.D., Kendrick, J., Russell, C., Christensen, J., Cunningham, J.E., Pearson, A.R., et al.: Effect of Molecular Size and Particle Shape on the Terahertz Absorption of a Homologous Series of Tetraalkylammonium Salts. Anal. Chem. 85, 7926-7934 (2013)

19. Allis, D.G., Zeitler, J.A., Taday, P.F., Korter, T.M.: Theoretical analysis of the solid-state terahertz spectrum of the high explosive RDX. Chem. Phys. Lett. 463, 84-89 (2008)

20. Pereverzev, A., Sewell, T.D., Thompson, D.L.: Molecular dynamics study of the pressure-dependent terahertz infrared absorption spectrum of $\alpha$ - and $\gamma$-RDX. J. Chem. Phys. 139, $-(2013)$

21. Pereverzev, A., Sewell, T.D.: Terahertz normal mode relaxation in pentaerythritol tetranitrate. J. Chem. Phys. 134, 014513 (2011)

22. Pereverzev, A., Sewell, T.D.: Terahertz spectrum and normal-mode relaxation in pentaerythritol tetranitrate: Effect of changes in bond-stretching force-field terms. J. Chem. Phys. 134, 244502 (2011)

23. Pereverzev, A., Sewell, T.D.: Molecular dynamics study of the effect of pressure on the terahertz-region infrared spectrum of crystalline pentaerythritol tetranitrate. Chem. Phys. Lett. 515, 32-36 (2011)

24. Pereverzev, A., Sewell, T.D.: Effect of vacancy defects on the terahertz spectrum of crystalline pentaerythritol tetranitrate. AIP Conf. Proc. 1426, 1187 (2012)

25. Pereverzev, A., Sewell, T.D., Thompson, D.L.: Calculation of anharmonic couplings and THz linewidths in crystalline PETN. J. Chem. Phys. 140, 104508 (2014)

26. Millar, D.I., Maynard-Casely, H.E., Allan, D.R., Cumming, A.S., Lennie, A.R., Mackay, A.J., et al.: Crystal engineering of energetic materials: Co-crystals of CL-20. CrystEngComm. 14, 3742-3749 (2012)

27. Hageman, J.A., Wehrens, R., de Gelder, R., Leo Meerts, W., Buydens, L.M.C.: Direct determination of molecular constants from rovibronic spectra with genetic algorithms. J. Chem. Phys. 113, 7955-7962 (2000)

28. N. Greenall, A. Valavanis, H.J. Desai, D.O. Acheampong, L.H. Li, J.E. Cunningham, et al.: Dataset Associated With The Development of a Semtex-H Simulant for Terahertz Spectroscopy. (2015)

29. Mitchell, M. An Introduction to Genetic Algorithms. MIT press, Cambridge, MA, USA (1998) 
30. MATLAB and the Global Optimisation Toolbox Release 2015b, The MathWorks, Inc., Natick, Massachusetts, United States., (2015)

31. Garnett, J.C.M.: Colours in Metal Glasses and in Metallic Films. Philosophical Transactions of the Royal Society of London. Series A, Containing Papers of a Mathematical or Physical Character. 203, 385-420 (1904)

32. Parrott, E.P., Zeitler, J.A., Gladden, L.F.: Accurate determination of optical coefficients from chemical samples using terahertz time-domain spectroscopy and effective medium theory. Opt. Lett. 34, 3722-3724 (2009)

33. Balan, E., Saitta, A.M., Mauri, F., Calas, G.: First-principles modeling of the infrared spectrum of kaolinite. Am. Mineral. 86, 1321-1330 (2001)

34. Kaushik, M., Ng, B., Fischer, B.M., Abbott, D.: Reduction of scattering effects in THz-TDS signals. IEEE Photonic. Tech. L. 24, 155-157 (2012)

35. Franz, M., Fischer, B.M., Walther, M.: The Christiansen effect in terahertz time-domain spectra of coarsegrained powders. Appl. Phys. Lett. 92, 021107-021107-021103 (2008)

36. Shen, Y., Taday, P., Pepper, M.: Elimination of scattering effects in spectral measurement of granulated materials using terahertz pulsed spectroscopy. Appl. Phys. Lett. 92, 051103 (2008)

37. Peng, J., Peng, S., Jiang, A., Wei, J., Li, C., Tan, J.: Asymmetric least squares for multiple spectra baseline correction. Anal. Chim. Acta. 683, 63-68 (2010)

38. O’Flynn, D., Desai, H., Reid, C.B., Christodoulou, C., Wilson, M.D., Veale, M.C., et al.: Identification of simulants for explosives using pixellated X-ray diffraction. Crime Science. 2, 4 (2013)

39. Desai, H.J. et al.: CAST explosives simulant classified patent on class of compounds referred to locally as 'Hemtex'. (2016) 